\title{
RETROBULBAR OPTIC NEURITIS AND CYSTOID MACULAR EDEMA AS A FIRST MANIFESTATION OF LYME DISEASE
}

\author{
Alexandra Tzoukeva ${ }^{1}$, Ara Kaprelyan', Diana Radkova ${ }^{2}$, Daniela Arabadjieva ${ }^{1}$ \\ ${ }^{1}$ Department of Neurology and Neuroscience, \\ ${ }^{2}$ Department of Infectious, Epidemiology and Tropical medicine \\ Medical University "Prof. Dr. Paraskev Stoyanov", Varna, Bulgaria
}

\begin{abstract}
Lyme disease is a multi-system disorder caused by the spirochete Borrelia burgdoferi. Eye manifestations are a rare involvement. We report two cases of patients who developed a retrobulbar optic neuritis and a cystoid macular edema as a sole clinical presenting in Lyme disease.

A 42-year-old female presented with left eye decreased visual acuity and painful ocular movement. Dilated funduscopy and neurological examination were normal. A diagnosis of left eye retrobulbar optic neuritis was made. The brain and spinal cord MRI showed typical lesions characteristic of MS. Serological tests for Lyme disease revealed positive results for IgM ELISA and Western blot. The possible serologically proven Lyme disease was diagnosed. Visual acuity returned to normal following a course of high-dose steroids and intravenous antibiotic, after by oral antibiotic. A 28 -year-old male presented with left eye blurred vision and intermittent blurring vision in his right eye. Dilated funduscopy revealed bilateral cystoid macular edema (left more than right). He was positive for Lyme serology, IgM ELISA and Western blot, and intravenous antibiotic therapy was commenced, followed by oral antibiotic. Visual acuity was restored and fuduscopy was normal.
\end{abstract}

In summary, although Lyme disease is an uncommon cause of these neuro-ophthalmic complications, our two patients highlight the importance of considering this disorder as a differential diagnosis and to initiate an early adequate therapy.

Keywords: Lyme disease, retrobulbar optic neuritis, cystoid macular edema

Address for correspondence:

Assoc. Prof. Alexandra Tzoukeva, $M D, P h D$,

Department of neurology and neuroscience

"Prof. D-r P. Stoyanov" Medical University of Varna,

55 M. Drinov str.

9002 Varna, Bulgaria,

phone: +359888648380

e-mail:al_tz@abv.bg

Received: November 13, 2014

Accepted: December 19, 2014

\section{INTRODUCTION}

Lyme disease, first identified in 1975 in Lyme, Connecticut, USA is caused by Borrelia burgdorferi. This tick-borne spirochetal disorder progresses in three stages and can cause symptoms in many body systems, including the eyes $(1,2)$. Ocular problems are infrequent involvements of Lyme disease that can appear at any stage of the disease and can take many different forms. Conjunctivitis is the most common ocular finding in stage I and II (3-6). Ophthalmic complications can be associated with neurologic in- 
Alexandra Tzoukeva, Ara Kaprelyan, Diana Radkova et al.

volvement, manifesting as ocular motility problems, optic neuritis, papilledema, and other possible ocular signs including uveitis, cystoid macular edema, scleritis, exudative retinal detachments (7-15). In the late stages of the Lyme borreliosis bilateral interstitial keratitis and symblepharon formation are described as characteristic findings. Enzyme linked immunosorbent assay (ELISA) test is used to detect antibody titers for the diagnosis of Lyme disease. Western blot is a qualitative test that is generally more sensitive and specific than ELISA. Although no test is 100\% accurate, Western blot test is helpful in differentiating a false positive result that can occur in patients with syphilis, tuberculosis, sarcoidosis, autoimmune disease (MS) or other neurologic disorders $(16,17)$. We report two cases of patients who developed a retrobulbar optic neuritis and a cystoid macular edema as a sole clinical presenting in Lyme disease.

\section{CASE REPORT I}

A 42-year-old female (DDR) was admitted to the Neuro-ophthalmology section of the Department of Neurology because of progressive decrease of visual acuity in the left eye and painful ocular movement during the last week. Neuro-ophthalmic examination revealed decreased visual acuity of 5/500 OS and $5 / 5 \mathrm{OD}$, left eye relative afferent pupillary defect and Ishihara testing with significant decrease in color vision. Dilated funduscopy was normal. A diagnosis of left eye retrobulbar optic neuritis was made. Neurological examination was normal. Standard hematological investigations, thyroid function tests, antinuclear antibodies, and syphilis serology were negative. Lumbar puncture was not performed. Cerebral and spinal cord Magnetic Resonance Imaging (MRI) showed typical lesions characteristic of MS. Serological examination of Neuromyelitis optica (NMO) - IgG/antiAQP4 antibody was negative. Intravenous high-dose Methylprednisolone was initiated for five days. After five days of treatment the visual acuity had improved to 5/6.3 OS. Further serological tests for Lyme disease showed a raised IgM ELISA titer and a positive result for Borrelia burgdorferi in the Western blot. The possible serologically proven Lyme disease was made in the absence of tick bites history and erythema migrans. A course of intravenous Medaxone and oral Cefzil was administered.
One month later visual acuity (5/5 OD and OS), pupillary light reaction, and color vision were normal.

\section{CASE REPORT II}

A 28-year-old male (VSI) presented to the Neuro-ophthalmologic section of the Department of Neurology with left eye blurred vision and intermittent blurring vision in his right eye for the last ten days. His medical history included a tick bite one year before but had never identified any rash. Neuro-ophthalmologic examination showed visual acuity of 5/6.3 OD and 5/50 OS. Dilated funduscopy revealed bilateral cystoid macular edema (left more than right), confirmed by fluorescein angiogram (FA) and OCT. Brain MRI was normal. Initial blood tests, including autoantibodies and syphilis serology were also negative. Serological tests for Lyme disease revealed a positive result for IgM ELISA and IgM Western blot. A diagnosis of Lyme disease was made. Antibiotic therapy with Medaxone (3.0 g daily) was commenced and oral Cefzil was administered. The patient responded subsequently to this antibiotic therapy. Six months after the treatment visual acuity was restored to 5/5 OD and OS, funduscopy was without macular edema.

\section{DISCUSSION}

Lyme disease is a multi-system disorder caused by the spirochete Borrelia burgdoferi, and has three clinical stages. Eye manifestations of Lyme disease are rare involvement $(1,2,4)$. Neuro-ophthalmological complications are most frequently seen in the late phases of the disorder, but some patients may not present all stages. As the serological tests lack sensitivity and specificity the etiological diagnosis of an isolated ocular manifestation as the presenting sign of Lyme borreliose must be based on medical history, clinical findings, and positive serological testing $(5,6,10,16,17)$. Retrobulbar optic neuritis as a sole sign of Lyme disease is rare, but it is most often caused by demyelinating disease. In our first case the patient with retrobulbar neuritis was with MRI typical lesions of MS, but with ELISA and Western blot positive results for Borrelia burgdorferi. In the recent literature the researchers discuss that patients with MS-like ocular symptoms probably had these symptoms due to MS and had also been exposed to the Borrelia bacterium. Based on the long-term follow- 
up of cases of isolated optic neuritis labeled as Lyme disease, because of the positive serology for Borrelia, there is an evidence for a later development of demyelination syndromes $(8,9,10,13,14,15)$. The second patient suffered from serologically proven Lyme disease with isolated cystoid macular edema as a first manifestation.

Current treatment protocols for Lyme disease include the use of oral, intramuscular, and intravenous antibiotic medications. A treatment protocol for severe neuro-ophthalmic or posterior ocular segment involvement in Lyme disease has not been established. In these cases therapeutic strategy should be based on the association of antibiotics and corticosteroids. A new course of therapy may be recommended to patients with relapsing ocular inflammation (18).

\section{CONCLUSION}

In this report we present two patients with retrobulbar optic neuritis and cystoid macular edema as a first manifestation of Lyme disease. In summary, although Lyme disease is an uncommon cause of these neuro-ophthalmic complications, our cases highlight the importance of this spirochetal disorder as a differential diagnosis and the necessity to initiate an early antibiotic therapy.

\section{REFERENCES}

1. Boye T. What kind of clinical, epidemiological, and biological data is essential for the diagnosis of lyme borreliosis? Dermatological and ophthalmological courses of Lyme borreliosis. Medecine et Maladies infectieuses, 2007; 37:175-188.

2. Winterkorn JM. Lyme disease: neurologic and ophthalmic manifestations. Surv Ophthalmol, 1990; 35:191-204.

3. Carvounis PE, Mehta AP, Geist CE. Orbital myositis associated with Borrelia burgdorferi (Lyme disease) infection. Ophthalmology, 2004; 111:1023-1028.

4. Kauffmann DJH, Wormser GP. Ocular Lyme disease: case report and review of the literature. $\mathrm{Br} \mathrm{J}$ Ophthalmol, 1990;74:325-327.

5. Mikkila HO, Seppala IJT, Viljanen MK, Peltomaa MP, Karma A. The expanding clinical spectrum of ocular Lyme borreliosis. Ophthalmology, 2000; 107:581-587.
6. Mora P, Carta A. Ocular manifestations of Lyme borreliosis in Europe. Int J Med Sci, 2009;6(3):124-125.

7. Amer R, Brannan S, Forrester JV. Inflammatory choroidal neovascular membrane in presumed ocular Lyme borreliosis. Acta Ophthalmol, 2009;87:346-348.

8. Blanc F, Ballonzoli L, Marcel C, De Martino S, Jaulhac B, de Seze J. Lyme optic neuritis. J Neurol Sci, 2010;295(1-2):117-119.

9. Burkhard C, Gleichmann M, Wilhelm H. Optic nerve lesion following neuroborreliosis: a case report. Eur J Ophthalmol, 2001;11:203-206.

10. Jacobson DM. Lyme disease and optic neuritis: long- term follow-up of seropositive patients. Neurology, 2003;60(5):881-882.

11. Jacobson DM, Frens DB. Pseudotumor cerebri syndrome associated with Lyme disease. Am J Ophthalmol, 1989;107:81- 82.

12. Krist $D$, Wenkel $H$. Posterior scleritis associated with Borrelia burgdorferi (Lyme disease) infection. Ophthalmology, 2002;109:143-145.

13. McVeigh $\mathrm{K}$, Vakros G. Case report: papillitis as the sole ocular sign in Lyme disease. Clin Ophthalmol, 2012;6:1093-1097.

14. Traisk F, Lindquist L. Optic nerve involvement in Lyme disease. Curr Opin Ophthalmol, 2012;23(6):485-490.

15. Todorovic L, Ibisevic M, Alajbegovic A, SuljicMehmedika E, Jurisic V. Bilateral retrobulbar optic neuritis as first signs of Lyme disease. Med Arh, 2008; 62(2):117-118.

16. Lindsay LR, Bernat $K$, Dibernaedo A. Laboratiry diagnostics for Lyme disease. CCDR, 2014;40:209-217.

17. Sibony P, Halperin J, Coyle PK, Patel K. Reactive Lyme serology in optic neuritis. J Neuroophthalmol, 2005;25(2):71-82.

18. Wright WF, Riedel DJ, Talwani R, Gilliam BL. Diagnosis and management of Lyme disease. Am Fam Physician, 2012; 85(11):1086-1093. 